\title{
Clearance of Thrombin from Circulation in Rabbits by High-affinity Binding Sites on Endothelium
}

\author{
POSSIBLE ROLE IN THE INACTIVATION OF THROMBIN BY
}

ANTITHROMBIN III

\author{
Pete Lollar and Whyte G. Owen, The Cardiovascular Center, \\ The Departments of Internal Medicine, Pathology and Biochemistry, \\ University of Iowa College of Medicine, Iowa City, Iowa 52242
}

\begin{abstract}
A B S T RA C T The clearance of ${ }^{125}$ I-thrombin and diisopropylphosphoryl-125I-thrombin (DIP-thrombin) from the circulation in rabbits was studied. When given either intraarterially or intravenously, DIP-thrombin, which is active-site blocked, was $\sim 90 \%$ cleared from the circulation by $1 \mathrm{~min}$, the time of earliest sampling, indicating a large first-pass effect. DIP-thrombin given intravenously is found predominantly in the lungs, whereas DIP-thrombin injected into the aortic arch is distributed diffusely in approximate proportion to the blood supply. Renal artery, femoral artery, ear artery, left atrium, and portal vein infusions demonstrate that kidney, muscle, ear, heart, and liver, respectively, can remove DIP-thrombin from the circulation. These data imply that the clearance of DIP-thrombin is not a function of a specific organ but of the vascular bed per se. The clearance of DIP-thrombin was reversible since injection of $0.5 \mathrm{mg}$ of unlabeled DIP-thrombin $10 \mathrm{~min}$ after the injection of a tracer dose of DIP-125I-thrombin resulted in the rapid reappearance of the DIP-125I-thrombin into the circulation. In addition, the clearance of DIP-thrombin was saturable, i.e., clearance of DIP-125Ithrombin was inhibited by unlabeled DIP-thrombin in a dose-dependent fashion. In vivo Scatchard analysis of the saturation of the clearance process demonstrated that DIP-thrombin can be removed by binding to highaffinity binding sites, since dissociation constants $\left(K_{\mathrm{D}}\right)$ of 10 and $13 \mathrm{nM}$ were obtained for human and bovine DIP-thrombin, respectively.
\end{abstract}

In contrast to DIP-thrombin, $\sim 75 \%$ of the radioac-

Dr. Lollar is the recipient of National Institutes of Health grant P-32-HL-07344-2. Dr. Owen is the recipient of Career Development Award K04 HL 00348-03 from the National Heart, Lung, and Blood Institute.

Received for publication 15 August 1979 and in revised form 11 August 1980. tivity associated with active thrombin remained in the circulation at $1 \mathrm{~min}$. By $10 \mathrm{~min} 55 \%$ of ${ }^{125} \mathrm{I}$-thrombin had been removed from the circulation, and essentially all of the radioactivity can be accounted for in the liver. Sodium dodecyl sulfate-polyacrylamide gel radioelectrophoresis of plasma samples taken after injection of ${ }^{125}$ I-thrombin demonstrated that all of the active thrombin was converted to covalent thrombin-antithrombin III complex by the time of initial sampling ( $30 \mathrm{~s})$. The in vitro conversion of ${ }^{125} \mathrm{I}$-thrombin to thrombin-antithrombin III complex was considerably slower $(50 \pm 5 \%$ conversion at $30 \mathrm{~s}$ ). The simultaneous injection of excess unlabeled DIP-thrombin inhibited the rate of formation of ${ }^{125}$ I-thrombin-antithrombin III complex formation in vivo (but not in vitro), which suggests that the binding of active thrombin to the high affinity binding sites is required for the rapid inactivation of thrombin in vivo.

We propose that $(a)$ thrombin in the circulation binds to active site-independent high-affinity binding sites on the endothelial cell surface; $(b)$ the inactivation of thrombin by antithrombin III is faster in vivo than in vitro because the high-affinity binding sites, present in a high concentration in the microcirculation, catalyze the reaction; $(c)$ thrombin-antithrombin III complexes are selectively removed by the liver.

\section{INTRODUCTION}

Thrombin has been shown to bind cultured human umbilical vein endothelium rapidly, reversibly, and with high affinity (1). This binding is active-site independent, i.e., diisopropylphosphoryl (DIP) thrombin ${ }^{1}$

${ }^{1}$ Abbreviations used in this paper: DIP, diisopropylphosphoryl; SDS-PAGRE, sodium dodecyl sulfate-polyacrylamide gel radioelectrophoresis. 
binds to endothelium in a manner indistinguishable from active thrombin.

In this paper we have studied the clearance of radiolabeled thrombin and DIP-thrombin from the circulation to test the hypothesis that the binding sites on endothelium play a role in the inactivation of circulating thrombin. We show that DIP-thrombin, which does not react with plasma substrates such as antithrombin III and fibrinogen, is cleared from the circulation by a high-affinity, reversible process. The clearance of active thrombin is more complex. We provide evidence that binding of active thrombin to endothelium in the microcirculation catalyzes the inactivation of thrombin by antithrombin III.

\section{METHODS}

Materials. Essentially fatty acid-free bovine serum albumin, Hepes, benzamidine, sulfopropyl- and QAE-Sephadex, and $\beta$-mercaptoethanol were purchased from Sigma Chemical Co., St. Louis, Mo. Diisopropylfluorophosphate (DFP) was purchased from Aldrich Chemical Co., Inc., Milwaukee, Wisc., and was diluted to $1 \mathrm{M}$ in dimethylformamide before use. Lactoperoxidase was purchased from Calbiochem-Behring Corp., American Hoechst Corp., San Diego, Calif. National Institutes of Health standard thrombin was obtained from the Bureau of Biologic's, Food and Drug Administration. Sodium dodecyl sulfate was purchased from BDH Chemicals, Ltd., Poole, England. Heparin, 1,000 U. S. U/ml, was purchased from A. H. Robins, Co., Richmond, Va. ${ }^{125} \mathrm{I}-\mathrm{Na}, 15 \mathrm{Ci} / \mu \mathrm{g}$, was purchased from Amersham Corp., Arlington Heights, Ill. Human ${ }^{131} \mathrm{I}$-albumin (Albumotope), $0.02 \mathrm{mCi} / \mathrm{mg}$, was obtained from E. R. Squibb \& Sons, Inc., Princeton, N. J. All other materials were reagent grade. Male New Zealand white rabbits weighing $1.5-2.5 \mathrm{~kg}$ were purchased from Morrison, Inc., West Liberty, Iowa.

Thrombin preparations. Rabbit, human, and bovine thrombins and human antithrombin III were prepared and assayed as described (2). All were essentially homogeneous as judged by sodium dodecyl sulfate polyacrylamide gel electrophoresis. The specific activities of bovine and human thrombins ranged from 2,400 to 2,600 and 2,700 to $3,500 \mathrm{U} / \mathrm{mg}$, respectively, based on an extinction coefficient $\left(\mathrm{E}_{280}^{1 \%}\right)$ of 19.5 for both preparations (3). For some experiments, thrombin was inactivated at pH 7.5 with $1 \mathrm{mM}$ DFP. An extinction coefficient of 5.7 $\left(\mathrm{E}_{280}^{1 \%}\right)$ was used for antithrombin III (4).

Radioiodination of thrombin. Human and rabbit thrombins were labeled with ${ }^{125} \mathrm{I}$ by the lactoperoxidase method of Thorell and Johanssen (5) as described, with the exception that the iodination was carried out in chloride-free buffers. The resulting product showed no loss of clotting activity for at least $1 \mathrm{wk}$ when stored at $4^{\circ} \mathrm{C}$. The incorporation of ${ }^{125} \mathrm{I}$ was determined by two independent methods: $(a)$ product specific activity method; the ratio of disintegrations per minute to clotting activity of the dialyzed ${ }^{125}$ I-thrombin preparation was determined and converted to moles ${ }^{125} \mathrm{I}$ per mole thrombin by using the specific activity of ${ }^{125} \mathrm{I}-\mathrm{Na}$ and specific clotting activity of the thrombin preparation (units per milligram). (b) Product precipitability method; the amount of ${ }^{125} \mathrm{I}$ incorporated per microgram of protein was determined by determining the disintegrations per minute of sulfosalicylic acid precipitability in the original reaction mixture and dividing by total micrograms of protein labeled. The number of ${ }^{125}$ I atoms per thrombin molecule was the same for both methods of determination $( \pm 15 \%)$ and ranged from 0.2 to 1.5 . This indicates that no fibrinogen clotting activity was lost by iodination. In addition, comparison of binding to cultured endothelial cells measured with increasing concentrations of ${ }^{125} \mathrm{I}$ thrombin with that determined by competing for ${ }^{125} \mathrm{I}$-thrombin binding with increasing concentrations of unlabeled thrombin showed no difference in affinity, thus indicating that the binding is not altered by iodination.

Experimental protocol. Rabbits were anesthetized with 50-200 $\mathrm{mg}$ ketamine hydrochloride i.m. and $3 \mathrm{~cm}^{3}$ of $2 \%$ lidocaine hydrochloride locally. Blood vessels were cannulated with blunted 19-gauge bufferfly needles after ligation proximal to the direction of injection. 1-ml tracer doses (l U. S. $\mathrm{U})$ of ${ }^{125} \mathrm{I}$ thrombin or DIP-125I-thrombin were injected into either a jugular vein, marginal ear vein, or aortic arch by way of a carotid artery. This dose of active thrombin is not thrombogenic in rabbits when assayed by the jugular vein stasis model of Gitel et al. (6). For blood volume determinations ${ }^{131} \mathrm{I}$-albumin was injected with the thrombin. Samples $(1 \mathrm{ml})$ were taken at intervals from the opposite carotid artery and counted in a Beckman DP 4000 gamma counter (Beckman Instruments, Inc., Fullerton, Calif.).

Determination of thrombin clearance. The amount of radiolabeled thrombin remaining in the circulation at any given time was determined as the percentage of injected dose as follows:

$$
\text { percent circulating }=\frac{\begin{array}{c}
\left(\mathrm{cpm}{ }^{125} \mathrm{I} / \mathrm{ml} \text { blood sample }\right) \\
\times(\text { blood volume }) \times 100
\end{array}}{\left(\mathrm{cpm}^{125} \mathrm{I} \text { injected }\right)} .
$$

The blood volume was calculated after extrapolating the blood disappearance curves of ${ }^{131} \mathrm{I}$-albumin to zero time by the equation: blood volume $=\left(\mathrm{cpm}^{131} \mathrm{I}\right.$ injected $) /\left(\mathrm{cpm}^{131} \mathrm{I} / \mathrm{ml}\right.$ of blood at time zero). Counts in the ${ }^{125} \mathrm{I}$ channel of the scintillation spectrometer were corrected for a $33 \%$ spill of ${ }^{131} \mathrm{I}$.

Determination of organ distribution of radioactivity. At the indicated times after injection of radiolabeled thrombin, the animal was killed with sodium pentobarbital and tissue samples were taken for determination of radioactivity. Small organs, such as brain, heart, and kidney, were counted in toto. Lung radioactivity was determined by taking a sample slice of known weight and multiplying by the organ weight to get total organ radioactivity. Liver radioactivity was determined by the same means as lung radioactivity except for experiments involving portal vein infusions. In these experiments the portal vein was infused with a tracer dose of DIP- ${ }^{125}$ I-thrombin over a 30-s interval. $90 \mathrm{~s}$ later the animal was killed, the liver was rapidly removed and homogenized in a Waring Blender (Waring Products Div., Hartford, Conn.) containing Hanks' balanced salt solution. The total organ radioactivity was calculated by using the homogenate volume and the radioactivity of $1-\mathrm{ml}$ samples. Selective infusions of the ear and kidney were performed by infusing $0.2 \mathrm{~cm}^{3}$ of a tracer dose of DIP${ }^{125}$ I-thrombin into an ear artery or renal artery with a 30 -gauge needle, followed by removal of the needle. In some experiments the left femoral artery was cannulated with a 20-gauge needle and DIP-125I-thrombin given over a 1 -min period. 2 min later the rabbit was killed and a leg muscle sample was taken. The fraction of radioactivity going to the heart was increased by opening the rabbit's chest, cannulating the left atrium with a 22-gauge needle and infusing ${ }^{125} \mathrm{I}$-DIP-thrombin over $30 \mathrm{~s}$ $90 \mathrm{~s}$ later the animal was killed and the distribution of radioactivity was determined.

Sodium dodecyl sulfate-polyacrylamide gel radioelectrophoresis (SDS-PAGRE) of rabbit plasma containing ${ }^{125} I$ thrombin. In some experiments $0.1 \mathrm{ml}$ of whole blood was withdrawn from the carotid artery cannula and added to 0.9 


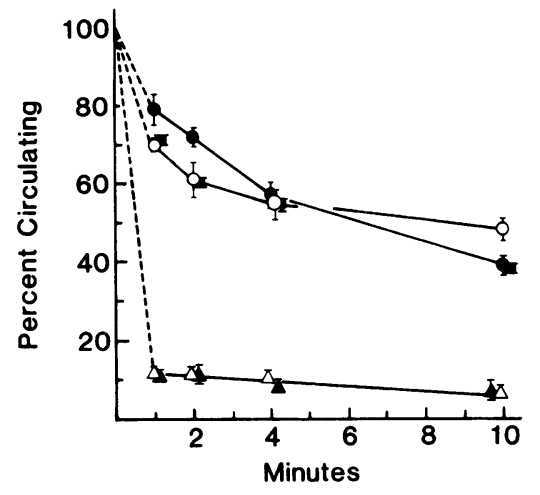

FIGURE 1 Clearance of ${ }^{125}$ I-thrombin and ${ }^{125}$ I-DIP-thrombin from the circulation. $1 \mathrm{U}$. S. U $\left(3-5 \times 10^{7} \mathrm{cpm}\right)$ of human ${ }^{125}$ I-thrombin (circles), DIP-125I-thrombin (triangles), or $1 \mathrm{ml}$ of autologous rabbit plasma previously mixed with $1 \mathrm{U}$. S. U ${ }^{125} I$-thrombin and 1 U. S. $U$ of heparin (squares) were injected either intravenously via the left internal jugular vein (closed symbols) or intraarterially into the aortic arch via the left carotid artery (open symbols) along with $1 \times 10^{5} \mathrm{cpm}$ of ${ }^{131} \mathrm{I}$-albumin. Samples $(1 \mathrm{ml})$ were taken from the carotid artery and the percent of injected dose remaining in the circulation was determined as described in Methods.

$\mathrm{ml}$ of $3.2 \%$ sodium citrate and $10 \mathrm{mM}$ benzamidine to inhibit the thrombin-antithrombin III reaction. The samples were centrifuged immediately for $15 \mathrm{~s}$ at $8,000 \mathrm{~g}$ in a Fisher model 59 high speed microcentrifuge (Fisher Scientific Co., Pittsburgh, Pa.). The diluted plasma supernates $(0.9 \mathrm{ml})$ were added to $0.1 \mathrm{ml} 10 \% \mathrm{SDS}$ and $14 \mathrm{mM} \beta$-merceptoethanol and heated in a $100^{\circ} \mathrm{C}$ water bath for $2 \mathrm{~min}$. A $0.1-\mathrm{ml}$ sample was then run on a $10 \%$ SDS polyacrylamide gel at $4 \mathrm{~mA} /$ tube according to the method of Laemmli (7). The amount of plasma protein loaded on the gel was $\sim 700 \mu \mathrm{g}$. The gels were sliced at $2-\mathrm{mm}$ intervals and radioactivity was determined.

Analysis of radioactivity in liver by SDS-PAGRE was performed by homogenizing a $1-\mathrm{g}$ liver slice in a Dounce homogenizer (Kontes Co., Vineland, N. J.) in $3.2 \%$ citrate- $10 \mathrm{mM}$ benzamidine, centrifuging at $8,000 \mathrm{~g}$ for $5 \mathrm{~min}$, and heating the supernate in $1 \%$ SDS, $14 \mathrm{mM} \beta$-mercaptoethanol in a boiling water bath for $2 \mathrm{~min}$. The sample was recentrifuged to remove debris and $25 \mu \mathrm{l}$ of the supernate was analyzed by electrophoresis.

Induction of thrombocytopenia in rabbits. Rabbits were given $900 \mathrm{rad}$ of total body irradiation over $20 \mathrm{~min}$. Platelet counts were determined manually by phase microscopy. A decline in platelet count was observed on the 4 th $d$. The experiment was performed on the 8th $\mathrm{d}$. The platelet counts were $40,000 \pm 30,000$ per $\mathrm{mm}^{3}$ in the five rabbits. The platelet count in eight normal controls was $320,000 \pm 50,000 / \mathrm{mm}^{3}$. Values refer to mean $\pm S D$.

\section{RESULTS}

Clearance of DIP-thrombin from the circulation. When rabbits were injected with DIP-125I-thrombin, either intravenously or intraarterially, $\sim 90 \%$ of the radioactivity was removed from the circulation by the time of the first sample (Fig. 1). The radioactivity remaining in the circulation appeared to be due to native
DIP-125I-thrombin since $(a)>95 \%$ of the radioactivity was precipitable by $10 \%$ sulfosalicylic acid at all times and $(b)$ SDS-PAGRE of plasma samples taken $10 \mathrm{~min}$ after injection demonstrated $90 \%$ of the radioactivity in the peak migrating with the $\alpha$-thrombin standard (Fig. 2). The small peak at slice 13 represents $\beta$-thrombin that was present in the sample before injection. All of the sample radioactivity was recovered in the gel. The clearance of rabbit DIP_125I-thrombin was identical to that of the human DIP-125I-thrombin (data not shown).

The distribution of DIP-125I-thrombin was determined by taking tissue samples immediately after the animals were killed. When given intravenously, 73\% of the DIP-thrombin was present in the lung (the first vascular bed it reached) at $10 \mathrm{~min}$ (Table I). When serial biopsies of the lung were taken, or when a gamma detector was placed over the thorax, the radioactivity in the lung slowly declined $\left(t_{1 / 2} \simeq 40 \mathrm{~min}\right)$, indicating redistribution of the DIP-thrombin. When DIP-thrombin was given intraarterially (caudad into the left carotid artery so that the solution was injected into the arch of the aorta) the radioactivity was distributed diffusely in rough proportion to the blood supply (Table I). Most of the radioactivity could not be accounted for in the major-organs; a large amount was in muscle, skin, and intestine, and was difficult to quantitate.

When ${ }^{125}$ I-DIP-thrombin and ${ }^{131} \mathrm{I}$-albumin were injected into the left renal artery and the animal killed after $4 \mathrm{~min}, 72 \%$ of the ${ }^{125}$ I remained in the kidney, whereas only $4.1 \%$ of the ${ }^{131} \mathrm{I}$-albumin remained (Table II). When the ear artery was injected, $25 \%$ of the ${ }^{125} \mathrm{I}$ was found in the ear as opposed to $2.8 \%$ of the ${ }^{131} \mathrm{I}$ - albu-

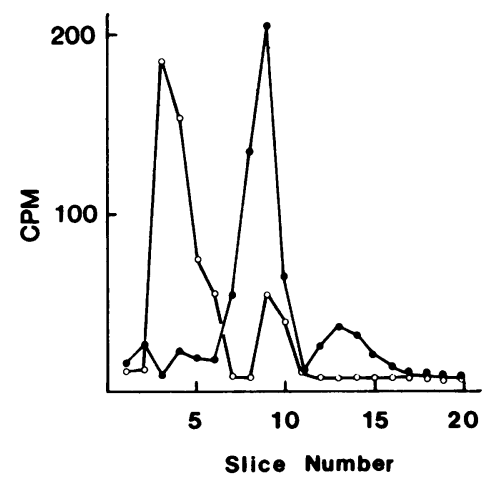

FIGURE 2 SDS-PAGRE of rabbit plasma containing radiolabeled thrombin. 1 U. S. U of human ${ }^{125}$ I-thrombin or 4 U. S. $U$ of DIP-125I-thrombin was injected into the left carotid artery, a $0.1-\mathrm{ml}$ blood sample was removed from the right carotid artery, after $30 \mathrm{~s}$ (active thrombin), or 10 min (DIP-thrombin) and processed for SDS-PAGRE as described in Methods. 0 , DIP-125I-thrombin in vivo, $O,{ }^{125} \mathrm{I}$ thrombin in vivo. When analyzed in parallel, authentic ${ }^{125} \mathrm{I}-$ thrombin-antithrombin III complex, prepared with purified antithrombin III, yielded a peak of ${ }^{125}$ I at slice number 3 , and ${ }^{125} \mathrm{I}$-DIP-thrombin gave a peak at slice number 9 . 
TABLE I

Organ Distribution of Injected Thrombin*

\begin{tabular}{|c|c|c|c|c|}
\hline & \multicolumn{2}{|c|}{ Active thrombin } & \multicolumn{2}{|c|}{ DIP-thrombin } \\
\hline & Intravenous & Intraarterial & Intravenous & Intraarterial \\
\hline & \multicolumn{4}{|c|}{$\%$ injected dose } \\
\hline Lung & $1.7 \pm 1.0$ & $1.4 \pm 2.0$ & $73 \pm 3$ & $20 \pm 3$ \\
\hline Liver & $46 \pm 8$ & $45 \pm 3$ & $8.5 \pm 1.0$ & $5.8 \pm 0.2$ \\
\hline Kidneys & $2.7 \pm 1.2$ & $2.9 \pm 0.85$ & $8.6 \pm 0.46$ & $7.4 \pm 4.1$ \\
\hline Heart & $0.3 \pm 0.1$ & $0.2 \pm 0.02$ & $0.50 \pm 0.01$ & $0.4 \pm 0.2$ \\
\hline Brain & $0.06 \pm 0.03$ & $0.05 \pm 0.02$ & $0.10 \pm 0.01$ & $0.4 \pm 0.1$ \\
\hline Blood & $48 \pm 3$ & $39 \pm 2$ & $6.5 \pm 0.30$ & $6.7 \pm 1.7$ \\
\hline \multirow[t]{2}{*}{ Unaccounted } & 1 & 12 & 3 & 60 \\
\hline & 100 & 100 & 100 & 100 \\
\hline
\end{tabular}

* Samples taken $10 \mathrm{~min}$ after injection of radiolabeled thrombin.

\$ Mean \pm SE, $n=3$ rabbits for active and DIP-thrombin injections.

min. The remainder of the ${ }^{125} I$ was found predominantly in the lung (Table II). Similarly, infusions of ${ }^{125}$ I-DIPthrombin into the hepatic portal vein, and left atrium resulted in the selective accumulation of ${ }^{125}$ I-DIPthrombin over the ${ }^{131} \mathrm{I}$-albumin control in liver and heart (Table II). When DIP-125I-thrombin was injected into the femoral artery and a leg muscle sample was taken, the percent ${ }^{125} \mathrm{I}$ injected/percent ${ }^{131} \mathrm{I}$ injected was $11 \pm 5$ (mean $\pm S D$ of three experiments). Thus the clearance of DIP-thrombin is not organ specific but rather a function of the vascular bed itself.

Clearance of active thrombin. The initial clearance of radioactivity associated with active thrombin is much less than that of DIP-thrombin (Fig. 1). At $1 \mathrm{~min}, 75 \%$ of the radioactivity is still present in the circulation, and after $10 \mathrm{~min} \sim 45 \%$ remains. The radioactivity present was almost entirely in a high molecular weight complex (Fig. 2). This high molecular weight complex is probably thrombin-antithrombin III complex since the complex $(a)$ forms in vitro at a much faster rate in the presence of heparin; (b) comigrates on SDSpolyacrylamide gels with purified thrombin-human antithrombin III complex; $(c)$ is apparently covalent since it is resistant to cleavage in hot SDS- $\beta$-mercaptoethanol; $(d)$ is active site specific, i.e., does not occur with DIP-thrombin. The small amount of radioactivity remaining in the thrombin region probably represents inactive thrombin, since heparinized plasma could convert only $90 \%$ of the thrombin into complex.

Analysis of the organ distribution of the radioactivity revealed that nearly all of the active thrombin removed from the blood was found in the liver (Table I), and all of the radioactivity was present in the form of high molecular weight complex.

When ${ }^{125}$ I-thrombin was added to heparinized autologous plasma and then injected the clearance curve was similar to that of the active thrombin injection (Fig. 1). Autopsy data also revealed that nearly all of the radioactivity which was cleared was present in the liver. These data indicate that thrombin-antithrombin III complex formation is rapid in vivo and that the complex is removed by the liver. The kinetics of formation of the complex are considered in further detail below.

Evidence that DIP-thrombin is cleared by high-affinity binding sites on the endothelial cell surface. The clearance of DIP-thrombin from the circulation is a reversible process. When a tracer dose of DIP- ${ }^{125}{ }^{25}-$ thrombin is followed by a large dose of unlabeled DIPthrombin, there is a rapid return into the circulation of $\sim 30 \%$ of the radioactivity, and the unlabeled DIPthrombin could be given $10 \mathrm{~min}$ after the injection of DIP-125I-thrombin and still result in the return of an

TABLE II

Selective Infusions of DIP-125I-Thrombin and ${ }^{131} I-A l b u m i n$

\begin{tabular}{|c|c|c|c|c|c|c|c|c|}
\hline & \multicolumn{2}{|c|}{ Ear } & \multicolumn{2}{|c|}{ Kidney } & \multicolumn{2}{|c|}{ Heart } & \multicolumn{2}{|c|}{ Liver } \\
\hline & ${ }^{125} \mathrm{I}$ & ${ }^{131}$ & 125I & ${ }^{13}{ }_{1}$ & ${ }^{125} I$ & ${ }^{131}$ & ${ }^{125} I$ & ${ }^{13} I$ \\
\hline & \multicolumn{8}{|c|}{$\%$ injected dose ${ }^{*}$} \\
\hline Ear & $25 \pm 4$ & $2.8 \pm 1$ & - & - & - & - & - & - \\
\hline Kidney & $4.3 \pm 1.0$ & - & $70 \pm 2$ & $4.1 \pm 0.5$ & - & - & - & - \\
\hline Lung & $45 \pm 5$ & - & - & - & $2.0 \pm 1.0$ & - & $4 \pm 2$ & - \\
\hline Heart & - & - & - & - & $2.8 \pm 0.8$ & $0.43 \pm 0.15$ & - & - \\
\hline Liver & - & - & $6.2 \pm 0.2$ & - & - & - & $52 \pm 5$ & $16 \pm 1$ \\
\hline Blood & $11 \pm 2$ & - & $10 \pm 2$ & - & $12 \pm 2$ & - & $15 \pm 5$ & - \\
\hline \multirow[t]{2}{*}{ Other } & 6 & - & 14 & - & 83 & - & 29 & - \\
\hline & 100 & - & 100 & - & 100 & - & 100 & - \\
\hline
\end{tabular}

* Mean and range, $n=2$ rabbits. 


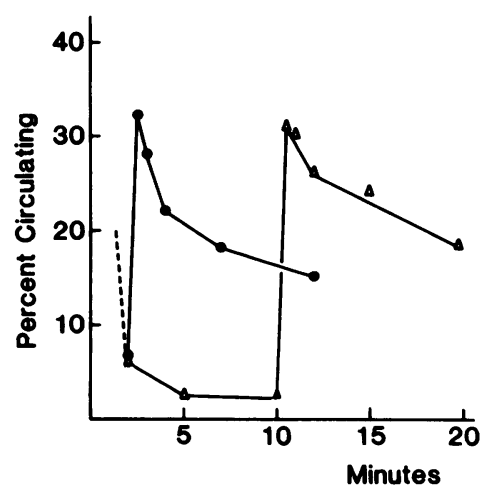

FIGURE 3 In vivo dissociation of bound DIP- ${ }^{125}$ I-thrombin. 1 U. S. U of human DIP-125I-thrombin was injected via a carotid artery along with ${ }^{131} \mathrm{I}$-albumin. 1-ml blood samples were taken at the indicated times and then a $0.5-\mathrm{mg}$ "chasing dose" of unlabeled DIP-thrombin was injected. Time of chasing dose: rabbit $1,2 \mathrm{~min}(O)$; rabbit $2,10 \mathrm{~min}(\triangle)$. The radioactivity is expressed as percent circulating as described in Methods.

identical amount of DIP-125I-thrombin (Fig. 3). We explain the observation that $<100 \%$ of the radioactivity returns to the circulation with a two-site binding model (see below), which, with the data in Fig. 4, predicts the amount of radioactivity returning to the circulation. The radioactivity that had returned to the circulation was analyzed by SDS-PAGRE. Greater than $90 \%$ of the radioactivity associated with the 2 -min and 10-min dissociation experiments was present in a peak migrating in the region of $\alpha$-thrombin. In addition, $100 \%$ of the radioactivity applied to the gel could be accounted for, indicating no degradation of the protein. This suggests that for at least $10 \mathrm{~min}$, circulating DIP-thrombin remains in an undisturbed equilibrium with a reversible pool of bound DIP-thrombin.

The clearance of DIP-thrombin is saturable because increasing amounts of unlabeled DIP-thrombin inhibit the clearance of tracer doses of DIP-125I-thrombin (Fig. 4A). Because of the reversibility and saturability of this process we propose a simple pharmacokinetic model for the clearance of DIP-thrombin. In this model DIP-thrombin present in the circulation (compartment 1) is in reversible equilibrium with binding sites on endothelium (compartment 2) and compartment 2 is saturable. The constants $k_{1}$ and $k_{2}$ represent the association and dissociation rate constants, respectively. In addition, DIP-thrombin is eliminated from the circulation by a process that is slow relative to $k_{1}$ and $k_{2}$. The rate constant of elimination, $k_{\mathrm{e}}, \sim 0.07 \mathrm{~min}^{-1}$ (Fig. 4A). This model is similar to the familiar two-compartment open model with a rapid preequilibrium phase, except that compartment 2 is saturable. This nonlinear pharmacokinetic model has been discussed by D. Santo et al. (8). A mathematical model for this process is:
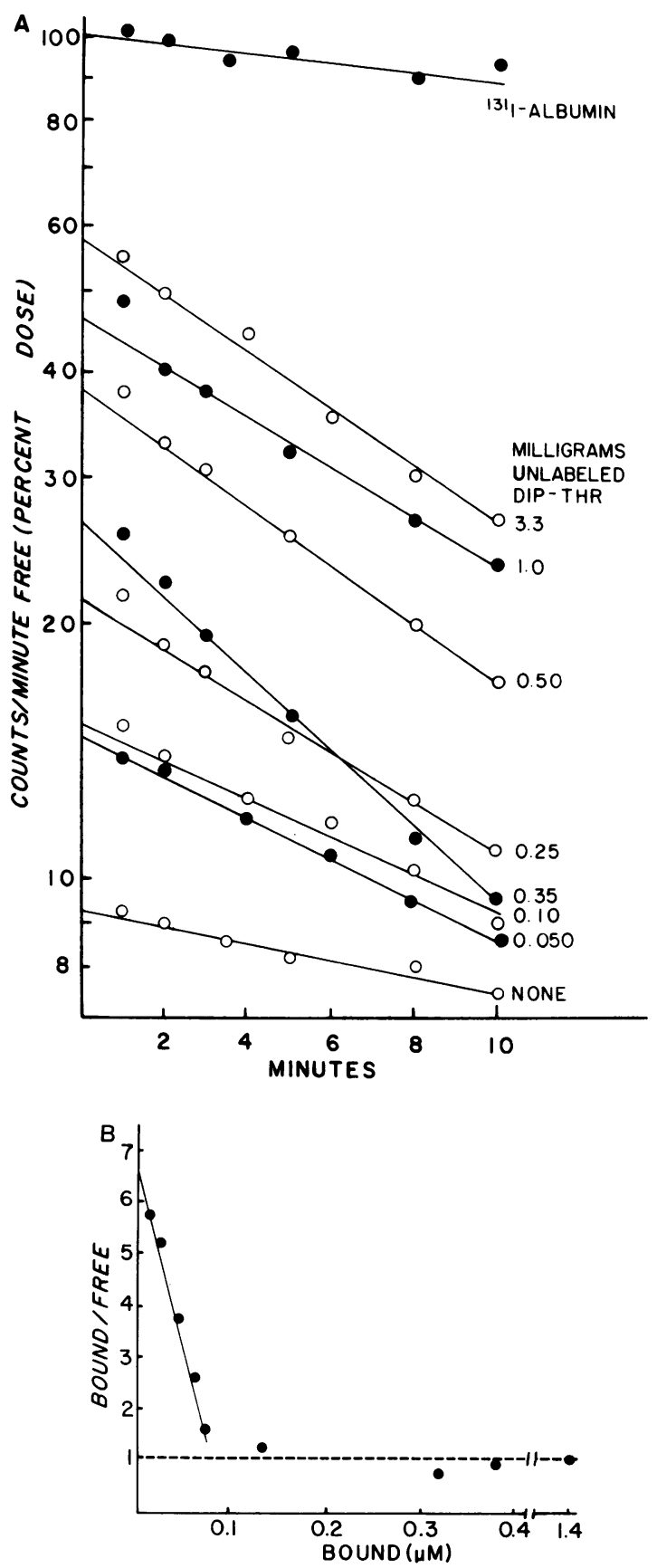

FIGURE 4 Saturation of thrombin clearance. (A) A tracer dose $\left(3-5 \times 10^{7} \mathrm{cpm}\right)$ of human DIP-125I-thrombin with $1 \times 10^{5} \mathrm{cpm}$ ${ }^{131}$ I-albumin were injected via a carotid artery along with a varied dose of unlabeled bovine DIP-thrombin. Samples were taken from the opposite carotid artery and analyzed as in Fig. 1. (B) Scatchard analysis of thrombin binding. The disappearance curves from Fig. 4A were extrapolated to zero time and the counts per minute at zero time were obtained. The thrombin free in the circulation was calculated from the specific activity of the total injected dose (counts per minute injected/ milligram injected) and expressed in concentration terms by dividing by the blood volume. The thrombin bound was then determined by subtracting free from the total dose injected. 
DIP-thrombin + binding sites

(compartment 1) (compartment 2)

$$
\begin{gathered}
\mathbf{k}_{1} \Downarrow \mathbf{k}_{2} \\
\text { DIP-thrombin } \\
\text { (compartment 2) } \\
\mathrm{k}_{\mathbf{e}} \\
\downarrow \\
\text { elimination, }
\end{gathered}
$$

which is equivalent to the expression for a reversible bimolecular interaction. By using this model, the amount of thrombin bound at equilibrium (i.e., cleared from the circulation) can be determined by extrapolating the clearance curves back to zero time, when no elimination has occurred. The data in Fig. 4A are then plotted according to the method of Scatchard (9) (Fig. 4B).

This results in a curvilinear plot. We propose that two classes of binding sites exist, one with high affinity and low capacity, the other with low affinity and high capacity. The low-affinity class of binding sites may represent the equivalent of "nonspecific" binding observed in in vitro binding systems. The dissociation constant $\left(K_{\mathrm{D}}\right)$ and concentration of the high-affinity binding sites $\left(R_{0}\right)$ were obtained from Fig. $4 B$ by the method of Klotz and Hunston (10). Values of 13 and 70 $\mathrm{nM}$ were obtained for the dissociation constant and concentration of binding sites, respectively. The experiment was also performed with human unlabeled DIP-thrombin. The $K_{\mathrm{D}}$ and $\mathrm{R}_{0}$ values obtained were 10 and $65 \mathrm{nM}$, respectively (data not shown) (Appendix). In this same experiment with human unlabeled DIP-thrombin, the effect of platelets on the clearance of DIP-thrombin was investigated, since platelets possess high-affinity binding sites for thrombin $(11,12)$. Rabbits were made thrombocytopenic by total body irradiation (Methods). There was little difference between the clearance curves of DIP-thrombin of normal rabbits and rabbits with an eightfold reduction of platelet count (data not shown) implying that platelets do not play an important role in this system.

The in vivo inactivation of thrombin. The timecourse of the in vivo inactivation of ${ }^{125}$ I-thrombin was followed by SDS-PAGRE as described in Methods and Fig. 2, and was compared to the inactivation of thrombin in whole blood in a siliconized tube. Fig. 5 (closed circles) demonstrates that $\sim 90 \%$ of the radioactivity is found in a high molecular weight complex at the time of initial sampling during the in vivo analysis. This represents the maximum amount of thrombin that is convertible to high molecular weight complex (Fig. 2 ); on the other hand, the in vitro inactivation of thrombin is slower (Fig. 5, open circles) and proceeds steadily over the time-course of the experiment. The amount of thrombin generated in the siliconized tube is much too small to compete for radio-labeled thrombin during



Frgure 5 Time-course analysis of inactivation of ${ }^{125} \mathrm{I}$-thrombin. In vitro analysis. $5 \mathrm{ml}$ of blood was drawn into a siliconized glass tube, $0.03 \mathrm{U}$. S. U of ${ }^{125} \mathrm{I}$-thrombin were added and the solution quickly mixed. $1 \mathrm{ml}$ of the blood was transferred to a tube containing $100 \lambda$ of $1 \mathrm{U} / \mathrm{ml}$ heparin. The tubes were incubated for the indicated times at $37^{\circ} \mathrm{C}$ and then $0.1 \mathrm{ml}$ was added to $0.9 \mathrm{ml} 3.2 \%$ citrate, $10 \mathrm{mM}$ benzamidine and processed for SDS-PAGRE as described in Fig. 2. The whole blood clotting time was greater than $5 \mathrm{~min}$ in all experiments. In vivo analysis. Rabbits were injected as in Fig. 2 . In both in vivo and in vitro analyses, radioactivity was present in two peaks corresponding to thrombin and thrombin-antithrombin III complex. The radioactivity present in the two peaks was summed, and percent thrombin remaining was calculated as the percentage of total radioactivity remaining in the peak corresponding to thrombin. In some cases, $2 \mathrm{mg}$ of DIP-thrombin was injected or $70 \mu \mathrm{g}$ of DIP-thrombin was added to $5 \mathrm{ml}$ in vitro along with the ${ }^{125} \mathrm{I}$-thrombin (excess DIP-thrombin). (O), in vivo, $\pm S D, n=4$ rabbits; ( $\square)$, in vivo plus excess DIP-thrombin, $n=3$; (O), in vitro, $n=4$; $(\square)$, in vitro plus excess DIP-thrombin; shaded area, mean $\pm S D, n=4$ of heparinized samples allowed to react $30 \mathrm{~s}$ in vitro.

the time-course of the reaction (13). The recovery of radioactivity from the gels was quantitative $(100 \pm 5 \%$, $n=8$ ); therefore, all of the radioactivity in whole blood could be accounted for in the two peaks.

The in vivo inactivation rate of thrombin was inhibited by the simultaneous injection of $2 \mathrm{mg}$ of unlabeled DIP-thrombin (Fig. 5, closed squares). At $30 \mathrm{~s}$, there is a statistically significant increase in the amount of free thrombin remaining $(P<0.05$, one-tailed $t$ test). By $60 \mathrm{~s}$ this difference has disappeared. This amount of DIP-thrombin is enough to saturate the high-affinity binding sites $95 \%$ without significantly occupying the low-affinity sites (Fig. 4B). A similar amount of DIPthrombin had no effect on the in vitro inactivation of thrombin (Fig. 5, open squares). These findings suggest that the initial binding of thrombin to the high-affinity, active site-independent sites on endothelium may be responsible for the difference in the in vivo and in vitro inactivation of thrombin by catalyzing the thrombinantithrombin III reaction. 


\section{DISCUSSION}

The stimulus for the experiments described in this paper came from the observation that thrombin binds with high affinity to cultured human endothelial cells. We reasoned that the concentration of binding sites would be much higher in the microcirculation than in vitro (given the same number of binding sites per cell) because of the much larger surface to volume ratio in the microcirculation compared to tissue culture dishes. The high concentration of binding sites might then be able to remove thrombin effectively from the circulation.

We found that DIP-thrombin, which can not interact with plasma substrates such as antithrombin III and fibrinogen, is, in fact, $\sim 90 \%$ cleared in its first pass through the circulation. Because the kinetics of DIPthrombin clearance are not complicated by interactions with known plasma substrates, we studied its clearance kinetics to determine whether high-affinity binding sites could be demonstrated in vivo and whether clearance is a function of the vascular bed per se.

The clearance of DIP-thrombin appears to be solely the function of the vascular bed since it is not specific for any organ. Thus, lung, liver, heart, kidney, ear, and muscle remove DIP-thrombin from the circulation (Table II and related results). The organ nonspecificity and the rapidity with which DIP-thrombin is both cleared and "chased" back into the circulation by unlabeled DIP-thrombin (Fig. 3) indicate that the vascular bed can bind DIP-thrombin and that the binding sites are in direct contact with circulating DIPthrombin, i.e., that they are on the endothelial cell surface. High-affinity binding is demonstrated by measuring the amount of DIP-thrombin cleared (i.e., bound) and the amount circulating (i.e., free) over several concentrations of DIP-thrombin (Fig. 4A), and then plotting the data according to the method of Scatchard (9) (Fig. 4B). We interpret these data as the result of two classes of binding sites. However, other causes of a concave upward curvilinear Scatchard plot (14) are not excluded. It does remain that at low concentrations of DIP-thrombin, high-affinity binding is seen. The dissociation constant and concentration of binding sites calculated for this process are $\sim 10$ and $70 \mathrm{nM}$, respectively. These calculations are valid only if $(a)$ the system equilibrates rapidly with respect to the elimination phase, $(b)$ binding is reversible, and $(c)$ the DIP-thrombin remains intact throughout the time-course of the experiment. Analysis by SDS-PAGRE of plasma samples taken 10 min after injection of DIP-125I-thrombin resulted in $100 \%$ recovery of radioactivity on the gel and predominantly in the peak of $\alpha$-thrombin, indicating the DIP-thrombin remains intact (Fig. 2).

Reversibility of DIP-thrombin binding was shown by chasing a small dose of DIP-125I-thrombin with $0.5 \mathrm{mg}$ of unlabeled DIP-thrombin at 2 and $10 \mathrm{~min}$. In both cases $\sim 30 \%$ of the injected radioactivity returned to the circulation (Fig. 3). This amount of reversibility is predicted by the two-site binding model. All of the bound counts do not return to the circulation because after injection of the relatively large dose of DIP-thrombin, the system re-equilibrates with significant binding to the low-affinity binding sites. Also, there is some re-equilibration of DIP-125I-thrombin with the high-affinity sites that the 0.5 -mg dose of DIP-thrombin does not completely saturate. These relationships are discussed quantitatively in the Appendix.

There are many similarities between thrombin binding in vivo and in tissue culture. Binding is very rapid (as opposed to many polypeptide-receptor interactions), rapidly reversible, and active site independent. The dissociation constant for the high affinity sites in vivo also is similar to the most recent estimate we have obtained with cultured cells, (15) 10 vs. $3 \mathrm{nM}$. Despite those similarities it is possible that the two phenomena are unrelated; further experiments are needed to resolve this issue.

The clearance of the radioactivity associated with active thrombin is slower (Fig. 1) and specific for the liver (Table I). In addition, it is associated with the formation of an apparently covalent, active site-specific high molecular weight complex. This radioactive complex forms in vitro at a much faster rate when heparin is added. This finding, along with the observation that the complex comigrates with purified thrombin-human antithrombin III on SDS polyacrylamide gels, and the knowledge that antithrombin III is the major inhibitor of thrombin in plasma (16), strongly support the conclusion that the high molecular weight complex is thrombin-antithrombin III complex.

The most interesting observation regarding the complex is that it forms much faster in vivo than in vitro. The in vitro formation of the complex has a half-life of formation of about $45 \mathrm{~s}$ (Fig. 5, open circles), which is similar to the half-life of thrombin in a physiological concentration of purified antithrombin III (17). On the other hand, the in vivo formation of complex is complete by the time of initial sampling (Fig. 5, closed circles). This raises the hypothesis that the difference between in vivo and in vitro rates of thrombin-antithrombin III complex formation is due to the binding of thrombin to the high-affinity binding sites, which then catalyze the thrombin-antithrombin III reaction. This is supported by the finding that a 2 -mg dose of unlabeled DIP-thrombin inhibits the rate of the thrombin-antithrombin reaction in vivo (Fig. 5 , closed squares) but does not inhibit the in vitro rate (Fig. 5, open squares). The concentration of ${ }^{125}$ I-thrombin in vitro was approximately equal to the initial in vivo concentration (Fig. 5, legend). The excess DIP-thrombin dos not inhibit the extent of the reaction however. This would be expected if unlabeled DIP-thrombin behaves as a competitive 
inhibitor of the binding-site catalyzed reaction, in much the same way as an enzyme-inhibitor reaction, where the rate of the reaction, but not the extent is inhibited.

An attractive possibility is that a heparin-like substance present on the endothelial cell surface is responsible for the binding of thrombin and catalysis of the thrombin-ATIII reaction. Among the small family of sulfated glycans known to catalyze the thrombinATIII reaction, heparan sulfate has been found in large quantities on cultured endothelial cells (18). Thrombin has been shown to bind to heparin with high affinity $\left(K_{\mathrm{D}} \simeq 1-100 \mathrm{nM}\right)$, although the binding characteristics have been only partially delineated (19-21). It has been shown that thrombin must bind to heparin for the accelerated reaction to occur $(19,20,22-26)$; furthermore, as with the in vivo reaction, active siteblocked thrombin inhibits the heparin-accelerated reaction, but not the heparin-independent rate (27).

Whether endothelium plays a general role in the clearance and inactivation of other clotting factors is an interesting possibility. If the high-affinity binding sites are due to a heparinoid, then other clotting factors that interact with heparin, such as Factors IX, X, XI, and plasmin, may be cleared by this mechanism. Also one may wonder whether deficiencies or inhibitors of the endothelial cell binding sites play a role in the pathogenesis of thrombotic disorders.

\section{APPENDIX}

We now may derive an expression predicting the amount of a small dose of radioactive ligand returned to the circulation after the injection of a large dose of unlabeled ligand, assuming two classes of binding sites exist for the ligand. After the injection of a large dose of unlabeled ligand, the system will relax toward a new equilibrium that now involves significant binding of ligand by the low-affinity, high capacity sites. Also, to the extent that the high-affinity, low capacity sites are not saturated, there will be some rebinding of radioactive ligand to the high-affinity sites.
The problem is to calculate the amount of free ligand $(F)$ relative to the total ligand $\left(\mathrm{L}_{0}\right)$ in the system because this will represent the fraction of free ligand, and therefore the fraction of free radioactivity, when the new equilibrium is reached.

From the conservation equation

$$
\mathrm{B}_{1}+\mathrm{B}_{2}+\mathrm{F}=\mathrm{L}_{0}
$$

where $B_{1}$ and $B_{2}$ are the amounts bound to the high- and low-affinity sites, respectively. The units are in concentration terms. Therefore

$$
\mathrm{F}=\mathrm{L}_{0}-\left(\mathrm{B}_{1}+\mathrm{B}_{2}\right)
$$

From the law of mass action,

$$
\mathrm{B}_{1}=\frac{\mathrm{R}_{0_{1}} \mathrm{~F}}{\mathrm{~F}+K_{\mathrm{D}_{1}}}
$$

and

$$
\mathrm{B}_{2}=\frac{\mathrm{R}_{0_{2}} \mathrm{~F}}{\mathrm{~F}+K_{\mathrm{D}_{2}}},
$$

where $\mathrm{R}_{\mathbf{0}_{1}}, \mathrm{R}_{\mathbf{0}_{2}}$ and $K_{\mathrm{D}_{1}}, K_{\mathrm{D}_{2}}$ refer to the concentration of binding sites and dissociation constants of the high- and low-affinity sites, respectively. Since the low-affinity sites are nonsaturable under the conditions used, $\mathrm{F} \ll K_{\mathrm{D}_{2}}$ and

$$
\mathrm{B}_{2}=\frac{\mathrm{R}_{0_{2}} \mathrm{~F}}{K_{\mathrm{D}_{2}}}
$$

From the Scatchard equation $\mathrm{R}_{0_{2}} / K_{\mathrm{D}_{2}}$ is the $y$ intercept of the Scatchard plot (9), and from Fig. $4 \mathrm{~B}$ this intercept $\simeq 1$ for the low-affinity site. When $K_{\mathrm{D}_{2}} \gg K_{\mathrm{D}_{1}}$ and $\mathrm{R}_{0,} \gg \mathrm{R}_{0,}$ this intercept is not altered by the high-affinity sites (10). Substituting $R_{0_{2}} /$ $K_{\mathrm{D}_{2}}=1$ into Eq. 5 and adding to Eq. 3,

$$
\mathrm{B}_{1}+\mathrm{B}_{2}=\frac{\mathrm{R}_{0_{1}} \mathrm{~F}}{\mathrm{~F}+K_{\mathrm{D}_{1}}}+\mathrm{F}
$$

substituting $\mathrm{E}_{1} .6$ into $\mathrm{E}_{1} .2$ leads to

$$
2 \mathrm{~F}^{2}+\left(2 K_{\mathrm{D}_{1}}+\mathrm{R}_{0_{1}}-\mathrm{L}_{0}\right) \mathrm{F}-\mathrm{L}_{0} K_{\mathrm{D}_{1}}=0
$$

Using the quadratic formula to solve for $F$,

$$
\mathrm{F}=\frac{\left(\mathrm{L}_{0}-2 K_{\mathrm{D}_{1}}-\mathrm{R}_{\mathrm{0}_{1}}\right)+\sqrt{\left(\mathrm{L}_{0}-2 K_{\mathrm{D}_{1}}-\mathrm{R}_{\mathrm{0}_{1}}\right)^{2}+8 \mathrm{~L}_{0} K_{\mathrm{D}_{1}}}}{4}
$$

Dividing by $\mathrm{L}_{0}$, gives the fraction of the injected dose that is free:

$$
\frac{\mathrm{F}}{\mathrm{L}_{0}}=\frac{\left(\mathrm{L}_{0}-2 K_{\mathrm{D}_{1}}-\mathrm{R}_{0_{1}}\right)+\sqrt{\left(\mathrm{L}_{0}-2 K_{\mathrm{D}_{1}}-\mathrm{R}_{0_{1}}\right)^{2}+8 \mathrm{~L}_{0} K_{\mathrm{D}_{1}}}}{4 \mathrm{~L}_{0}}
$$

Eq. 9 predicts the fraction of the injected dose returning to the circulation in terms of $\mathrm{L}_{0}$ and the two parameters estimated from the Scatchard plot, $K_{D_{1}}$ and $\mathrm{R}_{0_{1}}$. The total ligand concentration $\mathrm{L}_{0}$ is calculated from the mass of injected dose $(0.5 \mathrm{mg})$ and dividing by the blood volume of the rabbits in question in Fig. $3(120 \mathrm{ml}$ each) and the molecular weight of DIPthrombin $(37,400)$ and equals $110 \mathrm{nM}$. Using $K_{\mathrm{D}_{1}}=13 \mathrm{nM}$ and $\mathrm{R}_{0_{1}}=70 \mathrm{nM}$, and substituting into $\mathrm{E}_{\mathrm{1}} .8, \mathrm{~F} / \mathrm{L}_{0}=0.28$, or $28 \%$. This calculation, which depends only on equations of con- servation and mass action, agrees closely with the value obtained in Fig. 3.

\section{ACKNOWLEDGMENTS}

The authors wish to thank Doctors Christer Busch and John C. Hoak for helpful discussions. Radioiodinations were done in the laboratory of Dr. Barry Sherman. Ms. Rhonda Demuth gave excellent secretarial assistance. 
This work was supported, in part, by National Institutes of Health grant HL 14230-09 (Specialized Center for Research in Atherosclerosis).

\section{REFERENCES}

1. Awbrey, B. J., J. C. Hoak, and W. G. Owen. 1979. Binding of human thrombin to cultured human endothelial cells. J. Biol. Chem. 254: 4092-4095.

2. Owen, W. G. 1975. Evidence for the formation of an ester between thrombin and heparin cofactor. Biochim. Biophys. Acta. 405: 380-387.

3. Winzor, D. J., and H. A. Scheraga. 1964. Chemically reacting systems on Sephadex II. Molecular weights of monomers in rapid association equilibrium. J. Phys. Chem. 68: $338-343$.

4. Kurachi, K., G. Schmer, M. A. Hermodson, D. C. Teller, and E. W. Davie. 1976. Characterization of human, bovine, and horse antithrombin III. Biochemistry. 15: 368-372.

5. Thorell, J. I., and B. G. Johansson. 1971. Enzymatic iodination of polypeptides with ${ }^{125}$ I to high specific activity. Biochim. Biophys. Acta. 251: 363-369.

6. Gitel, S. N., R. C. Stephenson, and S. Wessler. 1977. In vitro and in vivo correlation of clotting protease activity: effect of heparin. Proc. Natl. Acad. Sci. U. S. A. 74: 328332.

7. Laemmli, U. K. 1970. Cleavage of structural proteins during the assembly of the head of bacterophage $\mathrm{T}_{4}$. Nature (Lond.). 227: 680-685.

8. Wagner, J. G. 1971. Biopharmaceutics and Relevant Pharmacokinetics. Hamilton Press, Hamilton, Ill. 302-318.

9. Scatchard, G. 1949. The attractions of proteins for small molecules and ions. Ann. N. Y. Acad. Sci. 51: 660-672.

10. Klotz, I. M., and D. L. Hunston. 1970. Properties of graphical representations of multiple classes of binding sites. Biochemistry. 10: 3065-3069.

11. Tollefsen, D. M., J. R. Feagler, and P. W. Majerus. 1974. The binding of thrombin to the surface of human platelets. J. Biol. Chem. 249: 2646-2651.

12. Martin, B. M., W. W. Wasiewski, J. W. Fenton II, and T. C. Detwiler. 1976. Equilibrium binding of thrombin to platelets. Biochemistry. 15: 4886-4893.

13. Shuman, M. A., and P. W. Majerus. 1976. The measurement of thrombin in clotting blood by radioimmunoassay. $J$. Clin. Invest. 58: 1249-1258.
14. Nichol, L. W. 1980. The interplay between ligand binding and protein acceptor association. In The Regulation of Coagulation, K. G. Mann, and F. B. Taylor, Jr., editors. Elsevier North-Holland, Inc., New York. 43-66.

15. Lollar, P., J. C. Hoak, and W. G. Owen. 1980. Binding of thrombin to cultured human endothelial cells. Non-equilibrium aspects. J. Biol. Chem. In press.

16. Hirsch, J. 1977. Hypercoagulability. Semin. Hematol. 14: 409-425. (Review.)

17. Jesty, J. 1979. Kinetics of formation and dissociation of the bovine thrombin-antithrombin III complex. J. Biol. Chem. 154: 10044-10059.

18. Buonassisi, V. 1973. Sulfated mucopolysaccharide synthesis and secretion in endothelial cell culture. Exp. Cell. Res. 76: 363-368.

19. Griffith, M. J. 1979. Covalent modification of human $\alpha-$ thrombin with pyridoxal-5-phosphate. J. Biol. Chem. 254: 3401-3406.

20. Smith, G. F. 1977. The heparin-thrombin complex in the mechanism of thrombin inactivation by heparin. Biochem. Biophys. Res. Commun. 77: 111-117.

21. Li, E. H. H., C. Orton, and R. D. Feinman. 1974. The interaction of thrombin and heparin: proflavin dye binding studies. Biochemistry. 13: 5012-5017.

22. Danishefsky, I., M. Ahrens, and S. Klein. 1977. Effect of heparin modification on its activity in enhancing the inhibition of thrombin by antithrombin III. Biochim. Biophys. Acta. 498: 215-222.

23. Hatton, M. W. C., and E. Regoeczi. 1977. The inactivation of thrombin and plasmin by antithrombin III in the presence of sepharose-heparin. Thromb. Res. 10: 645-660.

24. Machovich, R., G. Blasko, and L. A. Palos. 1975. Action of heparin on thrombin-antithrombin reaction. Biochim. Biophys. Acta. 379: 193-200.

25. Machovich, R. 1975. Mechanism of action of heparin through thrombin on blood coagulation. Biochim. Biophys. Acta. 412: 13-17.

26. Pomerantz, M. W., and W. G. Owen. 1978. A catalytic role for heparin. Evidence for a ternary complex of heparin cofactor, thrombin and heparin. Biochim. Biophys. Acta. 535: 66-77.

27. Griffith, M. J., H. S. Kingdon, and R. L. Lundblad. 1979. Inhibition of the heparin-antithrombin III/thrombin reaction by active site blocked-thrombin. Biochem. Biophys. Res. Commun. 87: 686-692. 